\title{
Circuit Health State Estimation via An Integrated Deep Neural Network
}

\author{
Ming Xiang ${ }^{1}$, Yigang $\mathrm{He}^{1 *}$, Hui Zhang ${ }^{1}$, Chaolong Zhang ${ }^{1}$, Zhaorong Zeng ${ }^{1}$, Baoran $\mathrm{An}^{2}$ \\ 1. School of Electrical Engineering and Automation, Wuhan University, Wuhan, 430072, China; \\ 2. Institute of Computer Application, China Academy of Engineering Physics, Mianyang, 621900, China \\ *corresponding author's email: yghe1221@whu.edu.cn
}

Received: August 5, 2020. Revised: September 29, 2020, Accepted: October 15, 2020. Published: October 21, 2020.

\begin{abstract}
The approaching of ubiquitous power internet of things is accelerating the industry to become more integrated and intricated. It is almost inevitable for a system to encounter failures during its whole life span. Thus, it is imperative to monitor the operating system from a system-level perspective to avoid potential catastrophes. Intuitively, inclusive prior knowledge is required for prognostics and health management (PHM). However, due to time-varying parameters and external conditions, the system is usually too complex to neatly fit into a prior-built model. This paper presents a novel pragmatic method, encompassing the convolutional autoencoder (CAE) and long short-term memory recurrent neural network (LSTM-RNN), to track the health state of a circuit. Briefly, the proposed method can be divided into two steps. First, degradation characteristics are extracted by using the time-domain features and CAE to prepare for the later health state estimation step. Then, the LSTM-RNN is used to finish the predictive process, i.e., to map the extracted abstract features to the health state. In addition, the degradation of a practical circuit considering the angular distance is discussed to quantify the health state of the circuit system. Furthermore, a case study based on that prognostics scheme is conducted to verify the proposed method. The comparison with other existing popular methods indicates the superiority of the proposed methodology.
\end{abstract}

Keywords-prognostics and health management (PHM), convolutional autoencoder (CAE), long short-term memory neural network (LSTM-RNN), angular distance.

\section{INTRODUCTION}

$\mathrm{T}$ HE impending Industry 4.0 is accelerating the smart manufacturing industry to a new era, thus making the system more and more integrated. In this way, the degradation of critical components within the system may spark unexpected disasters. For instance, three carriages of MTR Corps derailed in Hong Kong on September 17, 2019, the event that had warned people the inconceivable consequences of neglecting repairment, causing eight passengers injured and suspend of metro services. Therefore, prognostics and health monitoring (PHM) is vital for modern electronic systems.

Considering the methodologies behind the realization of PHM, they can be divided into three categories: model-based approaches, data-driven methods, and hybrid techniques. For the model-based approaches, if the parameters are precisely chosen, they can yield accurate estimations [1,2]. Luo et al. [3] proposed an interacting multiple model (IMM) for some undetectable failures. Nevertheless, because of the uncertainties from within like time-sensitive parameters and without, including variations in temperature, workload, comprehensive prior knowledge, and so forth, it is hard for that kind of method to be adopted. So the same as hybrid methods, since model-based approaches are considered.

On the other hand, the data-driven approaches can interpret the input data automatically and thus require no detailed knowledge for the system's structure. Under most scenarios, they follow the given steps to conduct PHM: first, necessary features are extracted; then, the method will match the health state of the system to the distilled features. Pan et al. conducted a real-time monitoring of machine health status with the help of dynamic fuzzy neural networks [4]. Babu et al. [5] estimated the remaining useful life (RUL) of the bearing on the basis of the regression method. Kiranyaz et al. [6] utilized adaptive 1-D convolutional neural networks (CNNs), which directly used the raw voltage and current data and avoided any feature extraction algorithm. Besides, CNN has been applied in a variety of fields [7-8]. However, each method has its own limitation: CNN, for instance, cannot comprehend the inner time-domain connections within the samples. An interesting integrated method, which inspires this study, was conducted by Gensler et al. [9]. A hybrid neural network, incorporating autoencoder (AE) and long short-term memory recurrent neural network (LSTM-RNN), was presented to predict solar power energy. The combination of the two networks took advantage of their respective unique merits: for the $\mathrm{AE}$, it could extract the features more precisely, and the LSTM-RNN, which is specially designed for time-series analysis, could effectively bridge the gap between the extracted features and the unknown RULs.

Broadly, PHM includes fault diagnosis and prognostics. Analog circuit fault diagnosis pinpoints the faulty components and, sometimes, provides correction solutions [10-12]. In comparison, circuit fault prognosis estimates the circuit 
performance in the future and possible failures. Some existing PHM researches for analog circuits focused on the degradation of specific elements like ceramic and embedded capacitors $[13,14]$, LED drivers [15]. However, in reality, it is not rare for us to encounter a degradation of multiple components in a given analog circuit. Consequently, manifesting the health state of a given circuit, when a degradation happens, is of immense significance. The Sallen-Key bandpass filter circuit [16], three-phase inverter bridge circuit [17], and an H-bridge circuit [18] are the usual testing analog circuits for PHM methods.

In this paper, a novel approach for analog circuit health state prognostics by using an integrated deep neural network, convolutional autoencoder- long short term memory network (CAE-LSTM), is proposed. Moreover, the health state of a circuit is decided by time-domain feature extractions and angular distance calculations of the measured signals. An MJ-level energy unit circuit, called the main power conditioning module (PCM), is employed to verify the effectiveness of the proposed method.

The paper is organized in the following order: Section II introduces the used health state estimation framework. Section III gives the backgrounds of PCM, simulation settings, results, and discussions. Finally, conclusions are drawn in Section IV.

\section{II.PROGNOSTICS FRAMEWORK}

The proposed method aims to estimate the health indicator (HI) of the system, which quantifies the health state of the system. First, a convolutional autoencoder is built and trained to extract the necessary characteristics. Then, LSTM layers will map the extracted features to the predicted HI.

\section{A. Convolutional Autoencoder}

Basically, a convolutional layer deals with images, and its operation procedure $c$ can be illustrated as follow:

$$
c_{(a, b)}=\sum_{m} \sum_{n} P_{(a+m, b+n)} K_{(m, n)}=\left(P^{*} K\right)_{(a, b)}
$$

where $P$ is two-dimensional data input, and $K$ is a onvolutional kernel.

However, with some modifications, it can also be employed for other signals. A strategy called 1-D CNN presented in Fig. 1 is used in this study to address this problem [19]. To be specific, the 1-D input signal is reconstructed into 2-D samples, including one dimension denotes the number of the time cycle of the signal, and the other one refers to the real sensory data.

As demonstrated by [20], a consecutive temporal sequence comprises denser information than a single data point. Thus time window slide, which reconstructs the original signals into discrete samples with the given size of the time window and the number of the selected features, is employed to represent the multidimensional temporal features. To be specific, it divides each signal sequence into a chosen length, then inserts these segmented sequences within the same time cycle period into

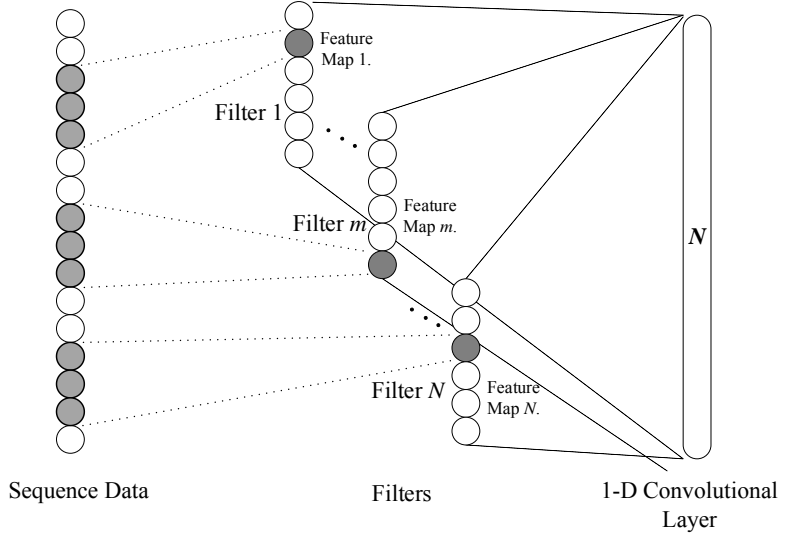

Fig. 1 A brief illustration of the 1-D CNN module

Table. 1 Settings of the CAE

\begin{tabular}{lcl}
\hline \multicolumn{1}{c}{ Layer } & Number & \multicolumn{1}{c}{ Filter Size } \\
\hline Convolutional layer & 2 & $\begin{array}{l}30 \text { filters of size }(1 \times 24) \text { and } 15 \\
\text { filters of size }(1 \times 6), \text { respectively }\end{array}$ \\
$\begin{array}{l}\text { Deconvolutional } \\
\text { layer }\end{array}$ & 2 & $\begin{array}{l}30 \text { filters of size }(24 \times 1) \text { and } 15 \\
\text { filters of size }(6 \times 1), \text { respectively } \\
\text { Max-pooling layer }\end{array}$ \\
$\begin{array}{lcl}(1 \times 12) \text { and }(1 \times 15), \text { respectively } \\
\text { Unsampling layer }\end{array}$ & 2 & $(1 \times 12)$ and $(1 \times 15)$, respectively \\
\hline
\end{tabular}

different channels of the fusion sample, like drawing a multi-channel image as the input for the network. In this study, the stride value and the size of a slide are set to be 1 and 15 cycles.

Generally, a convolutional autoencoder (CAE) consists of three types of layers [21]:

1) Convolutional layers: They are designed for input signal feature simplification, which projects the input signals to a higher-order space, thus obtains denser features.

2) Pooling layers: Pooling operations further eliminate redundant parameters to simplify the input.

3) Deconvolutional layers: similar to the convolutional layers, but with the inverse parameters, deconvolutional layers perform convolutional operations to generate the output with the same size as the corresponding convolutional layers.

The equations for $\mathrm{CAE}$ are formulated as follows:

$$
\left\{\begin{array}{l}
l=P\left(\operatorname{AF}\left(\operatorname{conv}(X)+b_{1}\right)\right) \\
\hat{X}=\operatorname{AG}\left(\text { de_conv }(l)+b_{2}\right) \\
\min L=D(X, \hat{X})+\Omega
\end{array}\right.
$$

where $P(\cdot), \operatorname{conv}(\cdot)$, de $\operatorname{conv}(\cdot)$ are the symbols for pooling, convolution, and deconvolution operations, respectively. $\mathrm{AF}(\cdot)$, $\mathrm{AG}(\cdot)$ denote the activation function for different elements. $b_{1}$, $b_{2}$ are the bias terms for the corresponding layers. $X$ and $\hat{X}$ are the original input and processed output for the $\mathrm{AE}$, respectively. $\mathrm{D}(\cdot)$ calculates the difference between $X$ and $\hat{X}, \Omega$ is the regularization term to reduce the overfitting problem. The goal 


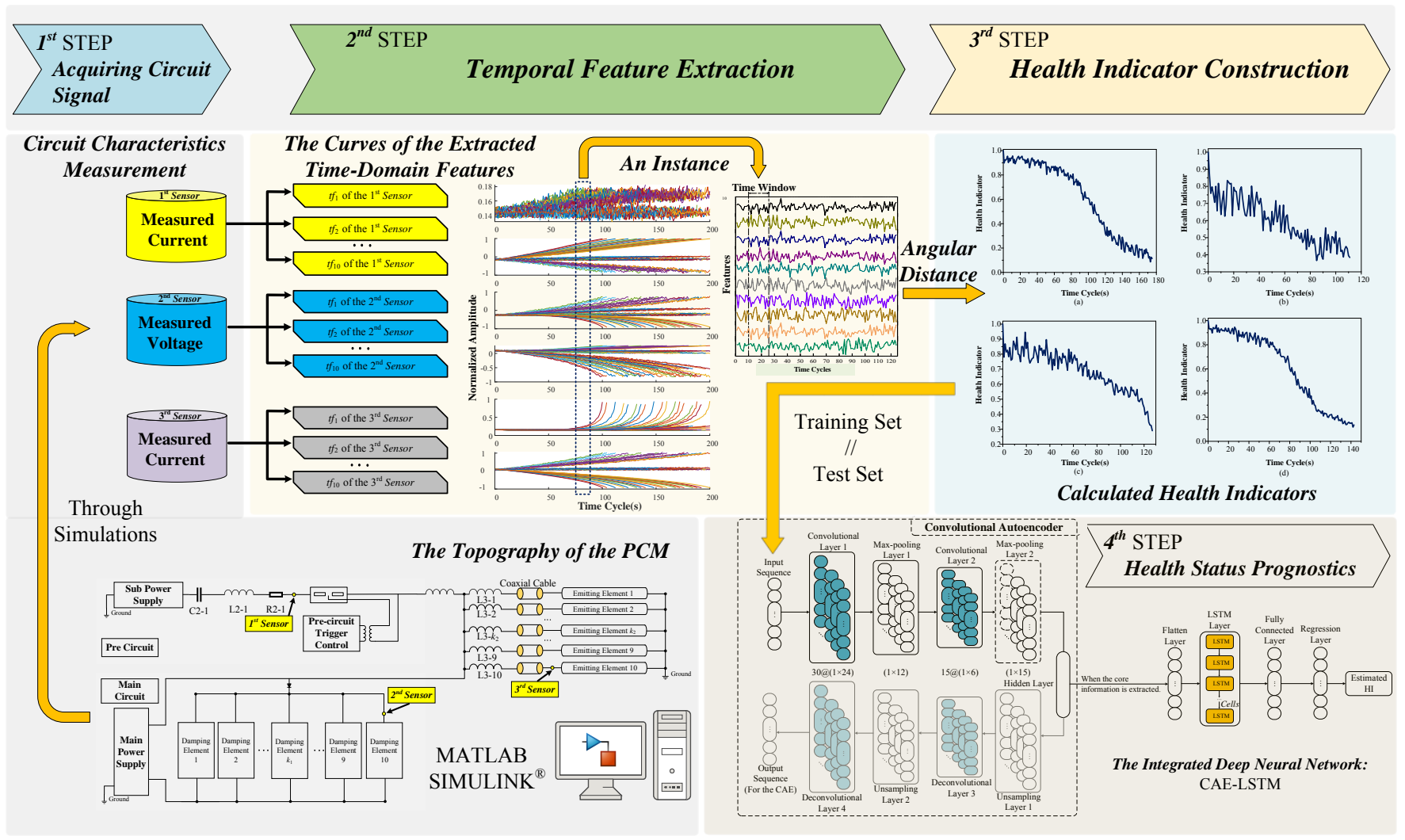

Fig. 2 The framework of the proposed prognostics method and the architecture of the integrated network

of CAE is to minimize the difference between the input and output, in other words, to minimize $L$.

Other settings are specified in Table 1. Once the training procedure is completed, the features in the hidden layer are extracted for LSTM regression.

\section{B. Long Short-Term Memory Recurrent Neural Network}

The LSTM-RNN, which is superior to the conventional RNN for its free from the problem of vanishing and exploding gradient, is born for time-series analysis [22].

The structure presented in Fig. 3 empowers the network to decide what data to be retained or deleted from its storage.

To manipulate and stable cell state, three different kinds of gates, including input, output, and forget gate, are used. The following equations illustrate the explicit implementation of cells at every step $i$.

$$
\left[\begin{array}{c}
\boldsymbol{i}_{\mathrm{i}} \\
\boldsymbol{o}_{\mathrm{i}} \\
\boldsymbol{f}_{\mathrm{i}}
\end{array}\right\rfloor=\sigma\left(\left\lfloor\begin{array}{lll}
w_{i x} & w_{i h} & b_{i} \\
w_{o x} & w_{o h} & b_{o} \\
w_{f x} & w_{f h} & b_{f}
\end{array}\right\rfloor \times\left\lfloor\begin{array}{c}
x_{i} \\
h_{i-1} \\
1
\end{array}\right\rfloor\right)
$$

where $w_{i x}, w_{o x}$, and $w_{f x}$ are weight coefficients of the input $x_{i}$ for the different gates of the cell, while $w_{i h}$, $w_{\text {oh }}$, and $w_{f h}$ are that of the former input variable $h_{i-1}$, respectively. $b_{\mathrm{i}}, b_{\mathrm{o}}$, and $b_{\mathrm{f}}$ denote bias of the input, the output, and the forget gate. Moreover, $\sigma$ is the symbol of the sigmoid function.

$$
\begin{aligned}
& z_{\mathrm{i}}=\varphi\left(w_{z x} x_{i}+w_{z h} h_{i-1}+b_{z}\right) \\
& \boldsymbol{c}_{\mathrm{i}}=z_{\mathrm{i}} \square \boldsymbol{i}_{\mathrm{i}}+\boldsymbol{c}_{\mathrm{i}-1} \square \boldsymbol{f}_{\mathrm{i}}
\end{aligned}
$$

$$
\boldsymbol{h}_{\mathrm{i}}=\varphi\left(c_{\mathrm{i}}\right) \square \boldsymbol{o}_{\mathrm{i}}
$$

where, likewise, $w_{z x}, w_{z h}$, and $b_{z}$ denote the weight coefficient of the input $x_{i}$, the former input variable $h_{i-1}$, and bias for the input node of the cell, respectively. $c_{\mathrm{i}}$ and $c_{\mathrm{i}-1}$ are values of the cell state at step i and i-1. In addition, $\varphi$ and $\square$ are the symbols of tanh function and pointwise multiplication.

Specifically, the function of each kind of gate is described as follows: (1) Whether the cell state will be updated and what information will be transited to the next step are determined by the input gate $\boldsymbol{i}_{\mathrm{i}}$; (2) The output gate $\boldsymbol{o}_{\mathrm{i}}$ filters information and modulates certain parts of the output cell state; (3) The forget gate $f_{\mathrm{i}}$ gathers data and deduces what data to be ruled out.

For this study, one LSTM layer with 50 cell structure is employed after the feature extraction process. In addition, the Adam algorithm is also considered to optimize the network parameters in the hope of better performance. The flow chart of the proposed schematic, including the architecture of the integrated network, is given in Fig. 2.

\section{CASE STUdy}

The proposed framework is verified through the degradation of a practical circuit, named the main power conditioning module (PCM). All the experiments are conducted on a computer with Intel Core i5-8400, 16 GB RAM, and simulated on MATLAB R2019a and Python 3.7.1 using the package "TensorFlow". Firstly, the degradation process of the PCM is described, ensued by the experimental settings of the 


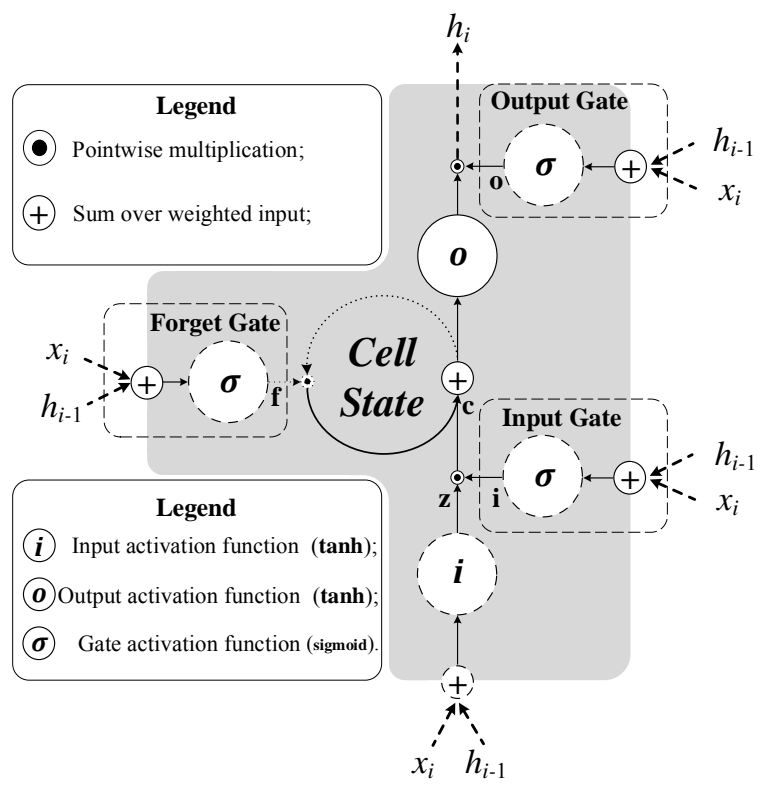

Fig. 2 Diagram of LSTM memory cell structure

framework. Then, assessment results are shown and discussed in the following section.

\section{A. Description of the PCM Degradation}

1) Backgrounds of The Main Power Conditioning Module

The MJ-level PCM, as presented in Fig. 4(a), is an indispensable power component of the SG-III energy facility, which is a prototype of the Chinese ignition project. Fig. 4(c) shows the framework of the facility. As the energy supply system of the facility is integrated, vast, and under comprehensive inspections, it is almost impossible for us to dive into the system and do some field studies, not to mention invasive experiments. Therefore, the degradation experiments are conducted via simulations.

Two sub circuits constitute the PCM. First, the main circuit comprises ten damping elements, each of which includes a resistor, inductor, and a capacitor with the respective values of $\mathrm{R}_{1-k 1}, \mathrm{~L}_{1-k 1}$, and $\mathrm{C}_{1-k 1}$. ( $k_{1}$ denotes the number of the damping element.) Then, in the pre circuit, there are capacitor $\mathrm{C}_{2-1}$, inductor $\mathrm{L}_{2-1}$, and resistor $\mathrm{R}_{2-1}$. It is worth mentioning that for the output part of the circuit, there are ten ballast inductors, i.e., $\mathrm{L}_{3-1} \sim \mathrm{L}_{3-10}$, and the relationship between the voltage and current of an emitting element is $\mathrm{I}=K \sqrt{\mathrm{U}}$. Additionally, the stimulated charging voltages of the pre and main power supply are $12 \mathrm{kV}$ and $23 \mathrm{kV}$, respectively. Besides, the $2^{\text {nd }}$ Sensor within the pre circuit monitors the voltage, the $1^{\text {st }}$ and $3^{\text {rd }}$ Sensor are used to formulate the current waveform.

There is a lag of $130 \mu \mathrm{s}$ between the trigger time of the $1^{\text {st }}$ switch and $2^{\text {nd }}$ switch. Specifically, after the main capacitors are discharged, the power flow would offer a high voltage power flow for the emitting elements with a duration of 480 930 $\mu \mathrm{s}$. As a result, it is evident in Fig. 4(b) that two discharge periods are separate, which prompts us to analyze them separately. The circuit characteristics are specified in Table 2.
Table. 2 The circuit characteristics

\begin{tabular}{lc}
\hline \multicolumn{1}{c}{ Description } & Set Value \\
\hline $\begin{array}{l}\text { The stimulated charging voltage of the } \\
\text { main circuit }\end{array}$ & $23 \mathrm{kV}$ \\
$\begin{array}{l}\text { The power flow duration of the emitting } \\
\text { element }\end{array}$ & $480 \sim 930 \mu \mathrm{s}$ \\
The current power flow duration of the & $460 \times(1 \pm 10 \%) \mu \mathrm{s}$ \\
main circuit & $1.2 \mathrm{MJ}$ \\
Maximum stored energy & $\geq 80 \%$ \\
$\begin{array}{l}\text { Energy transfer efficiency } \\
\text { The stimulated voltage of the pre circuit } \\
\text { The pulse width of the pre-operation } \\
\text { The current power flow duration of the } \\
\text { pre-operation }\end{array}$ & $12 \mathrm{kV}$ \\
\end{tabular}
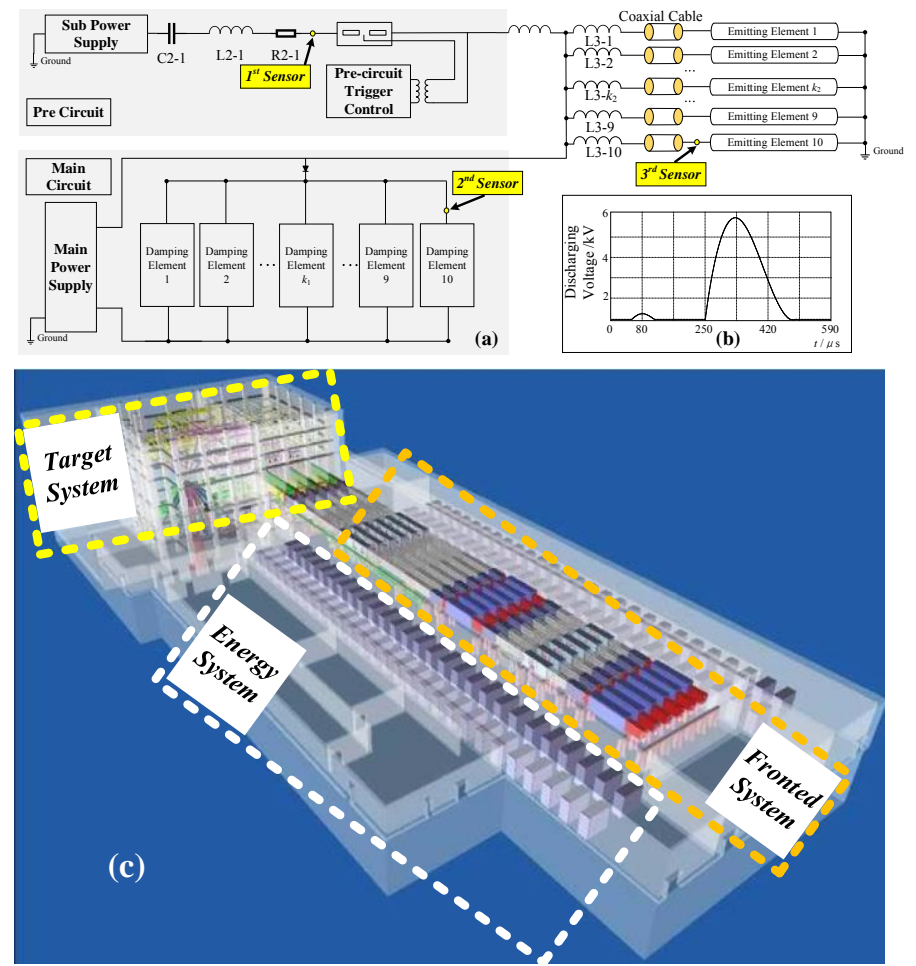

Fig. 4 (a) A diagram of the circuit, (b) A discharging voltage curve, (c) A bird's-eye view of the SG-III facility.

Table. 3 Illustration of time-domain features

\begin{tabular}{ll|ll}
\hline No. & Features & No. & Features \\
\hline 1 & $t f_{1}=\max \left(s_{t}\right)$ & 6 & $t f_{6}=\frac{t f_{5}^{2}}{t f_{4}^{3}}$ \\
2 & $t f_{2}=\frac{1}{N} \sum_{t=1}^{N} s_{t}$ & $t f_{7}=\frac{t f_{1}}{|\bar{s}|}$ \\
4 & $t f_{3}=\frac{1}{N} \sum_{t=1}^{N} s_{t}^{2}$ & & \\
4 & $t f_{4}=\sqrt{\frac{1}{N} \sum_{t=1}^{N}\left(s_{t}-\bar{s}\right)^{2}}$ & 9 & $t f_{1}$ \\
5 & $t f_{5}=\sqrt{\frac{1}{N} \sum_{t=1}^{N}\left(s_{t}-\bar{s}\right)^{3}}$ & 10 & $t f_{1}$ \\
\hline$f_{2}$ & $\frac{1}{N} \sum_{t=1}^{N}\left(s_{t}-\bar{s}\right)^{4}$ \\
\hline$f_{4}^{4}$
\end{tabular}


where $S_{t}$ is the value for the $t^{\text {th }}$ time point, $N$ denotes the total number of the sample, and they can be categorized into 2 groups: $t f_{1}-t f_{3}$ denote the energy and cumulative impacts of the signals. For $t f_{4}-t f_{10}$, they are the instances of statistical distribution.

\section{2) Degradation Settings}

\section{a) Time-domain Feature Extraction}

Time-domain features have already been applied in the realm of PHM [23] and proven potent. In this regard, to fully grasp the status of the degradation, ten time-domain [24] features presented in

Table. 3 are chosen.

\section{b) Parameter Setting}

It is considered that the circuit starts a degradation when the values of the critical electric components exceed beyond their tolerance range, i.e., $\pm 10 \%$ deviation from an element's nominal value [25], and reaches a complete failure once they go beyond the threshold, which is $60 \%$ deviation from its nominal value.

Since the probability for a single-component fault to happen is much higher than that for a multiple-component fault. Only different single-component faults are considered in this study.

Based on the degradation characteristics, it is assumed that the value of each selected element decreases directly and linearly. In this way, the value of the boundary is given as follow:

$$
\begin{aligned}
& \text { Value }_{\text {ft_up }}=(1-60 \%) \text { Value }_{\mathrm{n}}=0.4 \text { Value }_{\mathrm{n}} \\
& \text { Value }_{\mathrm{ft} \_ \text {low }}=(1-10 \%) \text { Value }_{\mathrm{n}}=0.9 \text { Value }_{\mathrm{n}}
\end{aligned}
$$

where $V_{\text {alue }}$ ftup refers to the upper limit value of the fault component, Value $\mathrm{ft}_{\mathrm{ft} \text { low }}$ refers to the lower limit value of the fault component, and $\bar{V}{ }^{2} \mathrm{lue}_{\mathrm{n}}$ denotes the nominal value of that element.

Furthermore, if the overall number of time cycles, namely Cycle $_{d}$, is decided, the stride value (Value stride $_{\text {e }}$ could be formulated as follow:

$$
\text { Value }_{\text {stride }}=\frac{\text { Value }_{\mathrm{n}}-\text { Value }_{\mathrm{ft}}}{\text { Cycle }_{\mathrm{d}}}
$$

Simulations with a Cycle d $_{\mathrm{d}}$ of 100 to 200 are conducted in the hope of emulating as many disparate scenarios as possible.

Table. 4 The Degradation Parameter Values

\begin{tabular}{ccccccc}
\hline No. Element & Cycle $_{\mathrm{d}}$ & Value $_{\mathrm{n}}$ & Value $_{\mathrm{ft} \_ \text {up }}$ & Value $_{\mathrm{ft} \_ \text {low }}$ & Value $_{\text {stride }}$ \\
\hline 1 & $\mathrm{~K}_{1-k^{*} \downarrow}$ & $100 \sim 200$ & 94.48 & 37.792 & 85.032 & $0.283 \sim 0.567$ \\
2 & $\mathrm{~L}_{1-k^{*} \downarrow}$ & $100 \sim 200$ & $140 \mu \mathrm{H}$ & $56 \mu \mathrm{H}$ & $126 \mu \mathrm{H}$ & $0.42 \mu \mathrm{H} \sim 0.82 \mu \mathrm{H}$ \\
3 & $\mathrm{~L}_{2-1} \downarrow$ & $100 \sim 200$ & $100 \mu \mathrm{H}$ & $40 \mu \mathrm{H}$ & $90 \mu \mathrm{H}$ & $0.3 \mu \mathrm{H} \sim 0.6 \mu \mathrm{H}$ \\
4 & $\mathrm{~L}_{3-k^{*} \downarrow}$ & $100 \sim 200$ & $30 \mu \mathrm{H}$ & $12 \mu \mathrm{F}$ & $27 \mu \mathrm{F}$ & $90 p \mathrm{H} \sim 180 p \mathrm{H}$ \\
5 & $\mathrm{C}_{1-i^{*} \downarrow}$ & $100 \sim 200$ & $87 \mu \mathrm{F}$ & $34.8 \mu \mathrm{F}$ & $78.3 \mu \mathrm{F}$ & $261 p \mathrm{~F} \sim 522 p \mathrm{~F}$ \\
6 & $\mathrm{C}_{2-1} \downarrow$ & $100 \sim 200$ & $14 \mu \mathrm{F}$ & $5.6 \mu \mathrm{F}$ & $12.6 \mu \mathrm{F}$ & $42 p \mathrm{~F} \sim 84 p \mathrm{~F}$ \\
\hline
\end{tabular}

$1 * k=1,2,3, \ldots, 10$. refers to the number of the element.

${ }^{2} \downarrow$ symbolizes a decrease in the values of the corresponding circuit element.
Besides, white Gaussian noise with a signal to noise ratio (SNR) of $40 \mathrm{~dB}$ is added in the raw signal to reflect the natural variations in parameters. In detail, Table 4 presents the degradation parameter settings.

According to the discussions above, the curves of the main and the sub charge circuit follow different patterns. Thus, the monitored data for every discharge period is divided into two segments to analyze two periods independently. As a result, 60 time-domain features are obtained. (Note that there are three sensors in the circuit.) For the proposed framework, a total of 156 samples are randomly divided into training and test groups with 109 and 47 specimens, respectively.

Additionally, a min-max strategy is used to normalize the extracted features, which assures the normalized features to distribute from -1 to 1 , i.e.,

$$
\overline{\boldsymbol{x}_{i}}=2 \frac{\boldsymbol{x}_{i}-\min \boldsymbol{x}_{i}}{\max \boldsymbol{x}_{i}-\min \boldsymbol{x}_{i}}-1
$$

where $\boldsymbol{x}_{i}$ is the time series of the $i^{\text {th }}$ sample, and $\bar{x}_{i}$ is the normalized time series.

\section{c) HI Construction}

Simply, the extracted temporal features cannot manifest the health state of the analog circuit themselves. Therefore, the revised angular distance is used to quantify the difference between the operation state and the original healthy state, aiming at constructing the HI, which is an indicator of the circuit's health state. In other words, the HI is the angular distance of two state vectors.

Take two vectors as instances, $\mathbf{x}_{1}=\left(\mathbf{x}_{1}^{(1)}, \mathbf{x}_{1}^{(2)}, \ldots, \mathbf{x}_{1}^{(\mathbf{n})}\right)$, which denotes the signal vector of healthy state, $\mathbf{x}_{2}=\left(\mathbf{x}_{2}^{(1)}, \mathbf{x}_{2}^{(2)}, \ldots, \mathbf{x}_{2}^{(\mathrm{n})}\right)$, the revised angular distance is presented as follow:

$$
\operatorname{dis}\left(\mathbf{x}_{1}, \mathbf{x}_{2}\right)=1-\frac{1}{\pi} \cdot \arccos \frac{\mathbf{x}_{1} \cdot \mathbf{x}_{2}}{\sqrt{\sum_{k=1}^{n}\left[\mathbf{x}_{1}^{(k)}\right]^{2} \times \sum_{k=1}^{n}\left[\mathbf{x}_{2}^{(k)}\right]^{2}}}
$$

It is worth noting that the distance distributes between $[0,1]$, consistent with the expected system health state.

\section{B. Health State Estimation}

1) Assessment Metrics

Two well-known metrics in PHM studies, i.e., scoring function and root mean squared error (RMSE), formulated by (10) and (11), respectively, are used in this study to evaluate the performance of the proposed method.

$$
R M S E=\sqrt{\frac{1}{N} \sum_{i=1}^{N} e_{i}^{2}}
$$

where $\mathrm{N}$ is the total number of the samples, and $e_{i}=\overline{\mathrm{HI}_{\mathrm{i}}}-\mathrm{HI}$, i.e., the predicted error for the $i^{\text {th }}$ sample.

$$
g=\sum_{i=1}^{N} g_{i}, \text { where } g_{i}=\left\{\begin{array}{l}
\exp \left(-\frac{e_{i}}{13}\right)-1, \text { for } e_{i}<0, \\
\exp \left(\frac{e_{i}}{10}\right)-1, \text { for } e_{i} \geq 0 .
\end{array}\right.
$$

where $g$ and $g_{i}$ denote the sum of the score and the score of the $i^{\text {th }}$ sample. 


\section{2) The HI Prediction Results}

Fig. 5 shows the estimation results of the proposed method for the degradation of PCM. The sharp decline in the beginning stage could be ascribed to the transition state of the component when a test began. Also, it can be observed in Fig. 5 that the predicted results could not track the curve of the test sample with $100 \%$ accuracy, and the failure inception prognostics are not perfectly matched with the original ones. However, the gaps between the calculated HIs and the predicted results are acceptable. For example, in No.106 Test, the predicted failure inception is Cycle 17, while the actual one is Cycle 20. Therefore, the prediction error, in this case, is 3 Cycles, which is relatively small compared with the range of the degradation simulation cycles. Additionally, two curves all follow the similar degradation trends, meaning that the proposed method could track the health state curve of the PCM effectively. It is also worth noting that the endpoints of the two curves are quite close, indicating the superiority of the study from another perspective.

Besides, the comparison between existing works, which is presented in Table. 5, indicates that the CAE-LSTM method outperforms other extant methodologies in terms of both the RMSE and the score. Specifically, consider deep CNN [24], the RMSE of the proposed method is approximately half of the deep CNN, while the score of the CAE-LSTM is lower than that of the $\mathrm{CNN}$ by $55 \%$. The satisfactory achievements that the method yields are possibly attributed to the combination of CAE and LSTM. Because the CAE is specialized in feature extraction to project the simple input into a higher-order space, whereas the LSTM-RNN is more experienced in time-series analysis to find the relationship within the discrete while inner connected time points. Therefore, the fused network can exploit the advantages of the two kinds of networks and circumvent some disadvantages. Besides, the results confirm the effectiveness of using such a framework to prognosticate the health state of the analog circuit.

\section{CONCLUSIONS}

In this paper, a novel data-driven PHM approach based on CAE and LSTM is proposed for a practical circuit, the PCM.

The study aims to achieve high predictive accuracy and put the method into industry applications. In addition, angular distance is applied in this study to quantify the health state of the system. The CAE-LSTM method is verified through the degradation test of the PCM. Comparisons between the proposed method and other popular methods prove that the method is superior regarding RMSE and the utilized score function.

The contributions of the paper can be summarized as follows:

1) A comprehensive degradation framework for a real analog circuit, PCM, is established using angular distance.

2) The integrated deep neural network, namely, CAE-LSTM, is built and utilized for prognostics. Also, the experimental results lend support to its effectiveness.

Future research lies in the following orientation: the integration of neural networks other than $\mathrm{CNN}$, and the integration of other optimization algorithms for better performance.

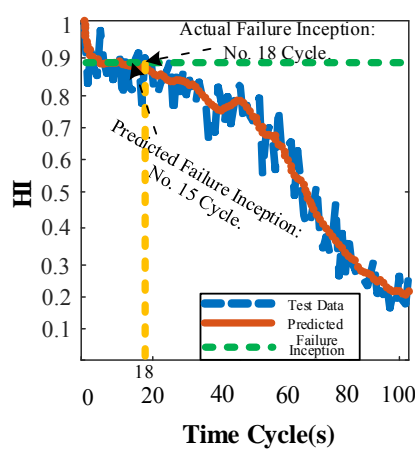

(a)

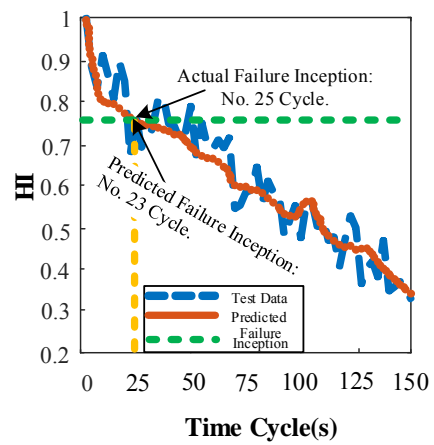

(c)

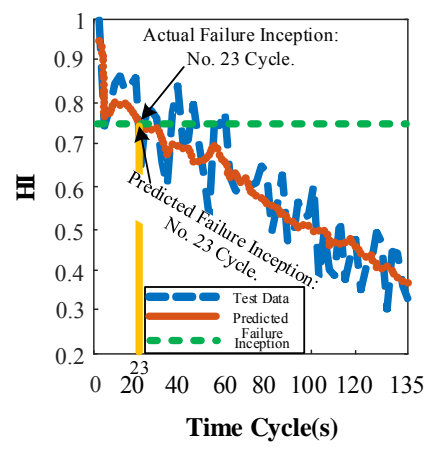

(b)

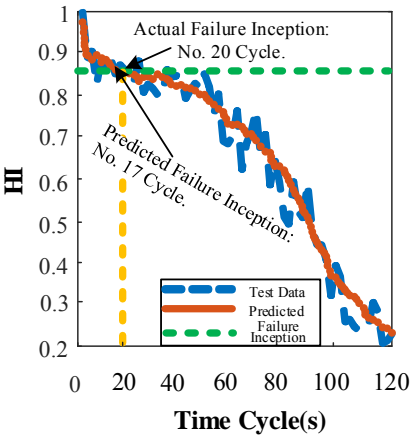

(d)
Fig. 5 HI estimation results for PCM. (a) No.31 Test, (b) No.62 Test, (c) No.81 Test, (d) No.106 Test

Table. 5 Comparison between existing works

\begin{tabular}{ccc} 
Methodologies & RMSE & Score \\
\hline CAE-LSTM & 0.057 & 17.10 \\
Deep CNN [26] & 0.103 & 26.43 \\
LSTM [27] & 0.083 & 18.65 \\
Support Vector Machine [28] & 0.193 & 98.63 \\
\hline
\end{tabular}

\section{REFERENCES}

[1] Y. Qian, R. Yan, and R. X. Gao, “A multi-time scale approach to remaining useful life prediction in rolling bearing," Mech. Syst. Signal Process., vol. 83, pp. 549-567, 2017.

[2] B. T. Thumati and S. Jagannathan, "A model-based fault-detection and prediction scheme for nonlinear multivariable discrete-time systems with asymptotic stability guarantees," IEEE Trans. Neural Networks, vol. 21, no. 3, pp. 404-423, 2010.

[3] J. Luo, K. R. Pattipati, L. Qiao, and S. Chigusa, "Model-based prognostic techniques applied to a suspension system," IEEE Trans. Syst. Man, Cybern. A Syst. Humans, vol. 38, no. 5, pp. 1156-1168, 2008.

[4] Y. Pan, M. J. Er, X. Li, H. Yu, and R. Gouriveau, "Machine health condition prediction via online dynamic fuzzy neural networks," Eng. Appl. Artif. Intell., vol. 35, pp. 105-113, 2014.

[5] G. S. Babu, P. Zhao, and X.-L. Li, "Deep convolutional neural network based regression approach for estimation of 
remaining useful life," in International conference on database systems for advanced applications, 2016, pp. 214-228.

[6] S. Kiranyaz, A. Gastli, L. Ben-Brahim, N. Al-Emadi, and M. Gabbouj, "Real-Time Fault Detection and Identification for MMC Using 1-D Convolutional Neural Networks," IEEE Trans. Ind. Electron., vol. 66, no. 11, pp. 8760-8771, Nov. 2019.

[7] D. Khoury and F. Keyrouz, A predictive convolutional neural network model for source-load forecasting in smart grids, WSEAS Trans. on Power Systems, vol. 14, pp. 181-189, 2019.

[8] A. Tashk, J. Herp, ES. Nadimi, Automatic Segmentation of Colorectal Polyps based on a Novel and Innovative Convolutional Neural Network Approach, WSEAS Trans. on Systems and Control, vol. 14, pp. 384-391, 2019.

[9] A. Gensler, J. Henze, B. Sick, and N. Raabe, "Deep Learning for solar power forecasting-An approach using AutoEncoder and LSTM Neural Networks," in 2016 IEEE international conference on systems, man, and cybernetics (SMC), 2016, pp. 2858-2865.

[10]C. Zhang, Y. He, R. Liu, L. Zhang, and S. Jiang, "An analog circuit fault diagnosis approach using DBN as a preprocessor," Int. J. Circuits, Syst. Signal Process., vol. 13, pp. 156-161, 2019.

[11] J. Shi, Y. Deng, Z. Wang, and Q. He, “A Combined Method for Analog Circuit Fault Diagnosis Based on Dependence Matrices and Intelligent Classifiers," IEEE Trans. Instrum. Meas., vol. 69, no. 3, pp. 782-793, Mar. 2020.

[12] W. He, Y. He, B. Li, and C. Zhang, "A Naive-Bayes-Based Fault Diagnosis Approach for Analog Circuit by Using Image-Oriented Feature Extraction and Selection Technique," IEEE Access, vol. 8, pp. 5065-5079, 2020.

[13] C. Bhargava, V. K. Banga, and Y. Singh, "An intelligent prognostic model for electrolytic capacitors health monitoring: A design of experiments approach," Adv. Mech. Eng., vol. 10, no. 10, Oct. 2018.

[14] M. A. Alam, M. H. Azarian, M. Osterman, and M. Pecht, "Prognostics of Failures in Embedded Planar Capacitors using Model-Based and Data-Driven Approaches," J. Intell. Mater. Syst. Struct., vol. 22, no. 12, pp. 1293-1304, Aug. 2011.

[15]L. Han and N. Narendran, "An Accelerated Test Method for Predicting the Useful Life of an LED Driver," IEEE Trans. Power Electron., vol. 26, no. 8, pp. 2249-2257, Aug. 2011.

[16] C. Zhang, Y. He, L. Yuan, and F. Deng, “A novel approach for analog circuit fault prognostics based on improved RVM," J. Electron. Test., vol. 30, no. 3, pp. 343-356, 2014.

[17]H. Huang and P. A. Mawby, "A lifetime estimation technique for voltage source inverters," IEEE Trans. Power Electron., vol. 28, no. 8, pp. 4113-4119, 2012.

[18] T. Dragicevic, P. Wheeler, and F. Blaabjerg, "Artificial Intelligence Aided Automated Design for Reliability of Power Electronic Systems," IEEE Trans. Power Electron., vol. 34, no. 8, pp. 7161-7171, Aug. 2019.
[19] X. Li, Q. Ding, and J.-Q. Sun, "Remaining useful life estimation in prognostics using deep convolution neural networks," Reliab. Eng. Syst. Saf., vol. 172, pp. 1-11, 2018.

[20] G. Varol, I. Laptev, and C. Schmid, "Long-Term Temporal Convolutions for Action Recognition," IEEE Trans.

Pattern Anal. Mach. Intell., vol. 40, no. 6, pp. 1510-1517, Jun. 2018.

[21] Y. Sun, B. Xue, M. Zhang, and G. G. Yen, “A Particle Swarm Optimization-Based Flexible Convolutional Autoencoder for Image Classification," IEEE Trans. Neural Networks Learn. Syst., vol. 30, no. 8, pp. 2295-2309, Aug. 2019.

[22] H. Miao, B. Li, C. Sun, and J. Liu, "Joint Learning of Degradation Assessment and RUL Prediction for Aeroengines via Dual-Task Deep LSTM Networks," IEEE Trans. Ind. Informatics, vol. 15, no. 9, pp. 5023-5032, Sep. 2019.

[23] Y. Cheng, H. Zhu, J. Wu, and X. Shao, "Machine Health Monitoring Using Adaptive Kernel Spectral Clustering and Deep Long Short-Term Memory Recurrent Neural Networks," IEEE Trans. Ind. Informatics, vol. 15, no. 2, pp. 987-997, Feb. 2019.

[24] A. Hanif, Y. Yu, D. DeVoto, and F. Khan, “A Comprehensive Review Toward the State-of-the-Art in Failure and Lifetime Predictions of Power Electronic Devices," IEEE Trans. Power Electron., vol. 34, no. 5, pp. 4729-4746, May 2019.

[25]Z. Liu, T. Liu, J. Han, S. Bu, X. Tang, and M. Pecht, "Signal Model-Based Fault Coding for Diagnostics and Prognostics of Analog Electronic Circuits," IEEE Trans. Ind. Electron., vol. 64, no. 1, pp. 605-614, Jan. 2017.

[26]B. Yang, R. Liu, and E. Zio, "Remaining Useful Life Prediction Based on a Double-Convolutional Neural Network Architecture," IEEE Trans. Ind. Electron., vol. 66, no. 12, pp. 9521-9530, 2019.

[27] C.-S. Hsu and J.-R. Jiang, "Remaining useful life estimation using long short-term memory deep learning," in 2018 IEEE International Conference on Applied System Invention, 2018, pp. 58-61.

[28] C. Louen, S. X. Ding, and C. Kandler, "A new framework for remaining useful life estimation using Support Vector Machine classifier," in 2013 Conference on Control and Fault-Tolerant Systems, 2013, pp. 228-233.

Ming Xiang is now pursuing a master degree in electrical engineering at School of Electrical Engineering and Automation, Wuhan University. He received the bachelor degree in electrical engineering and its automation from Wuhan University in 2018. His current research interests include fault diagnosis and prognostics of analog circuits.

Yigang He received the M.Sc. degree in electrical engineering from Hunan University in 1992 and Ph.D. degree from Xi'an Jiaotong University in 1996. He is currently a professor at School of Electrical Engineering and Automation, Wuhan University. He is one of winners of National Distinguished Young Scientists Foundation. His research interests are in the 
areas of circuit theory and its applications, testing and fault diagnosis of analog and mixed-signal circuits, smart grid, radio frequency identification technology, and intelligent signal processing.

\section{Sources of funding for research presented in a scientific article or scientific article itself}

Report potential sources of funding if there is any

This work was supported in part by the National Natural Science Foundation of China under Grant No. 51977153, 51977161, 51577046, the State Key Program of National Natural Science Foundation of China under Grant No. 51637004, the national key research and development plan "important scientific instruments and equipment development" Grant No.2016YFF0102200, Equipment research project in advance Grant No.41402040301.

\section{Creative Commons Attribution License 4.0 (Attribution 4.0 International, CC BY 4.0)}

This article is published under the terms of the Creative Commons Attribution License 4.0

https://creativecommons.org/licenses/by/4.0/deed.en_US 